\title{
PERSPECTIVES OF SUSTAINABLE DEVELOPMENT AND SUSTAINABLE BUSINESS MODELS IN THE POST-COVID ERA
}

\author{
IA NATSVLISHVILI \\ PhD in Economics, Associate Professor \\ Ivane Javakhishvili Tbilisi State University, Georgia \\ ia.natsvlishvili@tsu.ge
}

\begin{abstract}
The article assesses the perspectives of achieving sustainable development goals and implementation of sustainable business models in Post-Covid era. Challenges created by the novel corona virus (Covid-19) pandemic have been analyzed in the context of sustainable development using desk research method and qualitative content analysis. Pandemic crisis gradually develops into a «triple wave» crisis: 1) pandemic shock and public health crisis; 2) a stronger shock of economic global mega-recession, 3) an even more powerful ecological crisis caused by climate change and declining biodiversity might be emerge.

According to the Organization for Economic Cooperation and Development (OECD) the total «lockdown» of economy caused by pandemic leads to a reduction of almost one-third of the gross domestic product (GDP) in the worldıs leading economies. Each month of economic "lockdown" causes a $2 \%$ loss of annual GDP. If some sectors of the economy can only be partially "locked down", then the initial reduction in GDP in most leading economies will be $20 \%-25 \%$. According to the World Bank pandemic and "lockdown" of world's leading economies could push to extreme poverty more than 60 million people and eliminate all the results achieved in the past years in poverty eradication.

Achieving sustainable development goals and implementation of sustainable business models might stay away from attention. In the future the frequency of pandemics will increase if humanity does not stop destroying nature. It is known that among mammals and water birds 1.7 million unknown viruses are inhabited that could be transmitted to human beings. Unrestrained deforestation, agrarian expansion, and infrastructure creation bring humans closer to these mammals and birds.

The pandemic situation is causing changes in the socio-political context. There are signs of politicization in the fight against corona virus-phenomena called infodemia and covidism. We observe excessive bans introduced by governments, restrictions on international trade, calls for protectionism, propaganda of nationalism, attempts to introduce authoritarianism, violation of fundamental human rights, attempts to spread mass fear and hysteria through the media.

The article evaluates two alternative scenarios for socio-economic development of post-pandemic period. In the current global mega-recession markets are changing radically, in the face of growing uncertainty firms are trying to adapt to new conditions. On the front line the «big» state appears, which acts as a leader in dealing with the pandemic and restoring the economy. In the Post-Covid era, a «new normal» situation is expected to emerge, which can develop in two possible directions. Undesirable direction is reduction of public services, ecological crisis caused by climate change, nationalism, autocracy. The preferred direction of development is more internationalization, new paradigms of business management, investments oriented on sustainable development, implementation of sustainable business modes, a new green deal and democracy. In order to reduce the risks of future pandemics, in the Post-Covid period a stimulus policy should be concentrated on the protection of human life and healthcare, provision of livelihoods, nature conservation and protection.
\end{abstract}

KEYWORDS: COVID-19, SUSTAINABLE DEVELOPMENT, SUSTAINABLE BUSINESS MODELS, ECONOMIC RESECTION, PANDEMICS, COVIDISM, INFODEMIA.

For citation: Natsvlishvili, I., (2020). Perspectives of Sustainable Development and Sustainable Business Models in the PostCovid Era. Globalization and Business, 10. 227-232. (In Georgian) https://doi.org/10.35945/gb.2020.10.029 


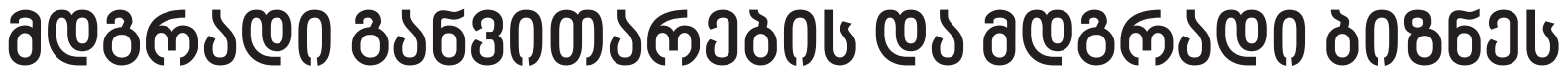

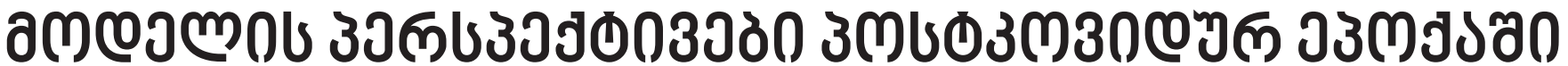

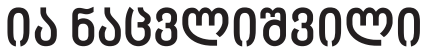

эзмбмдаз

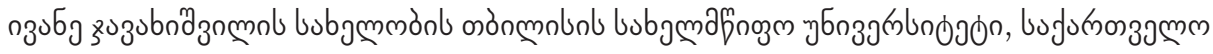

ia.natsvlishvili@tsu.ge

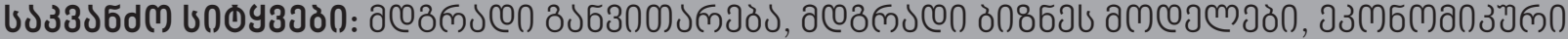

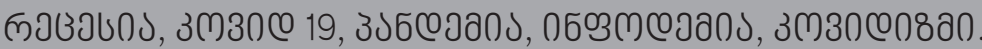

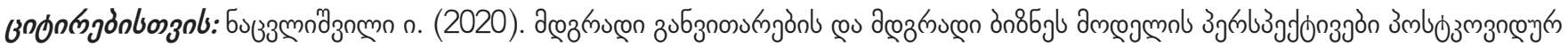

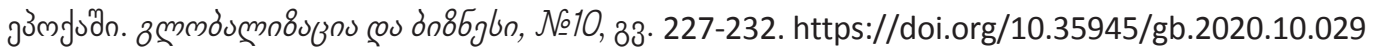

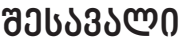

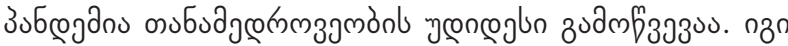

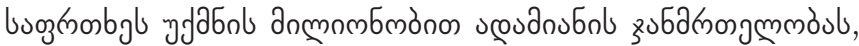

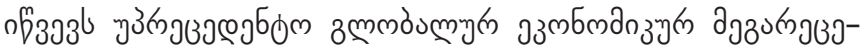

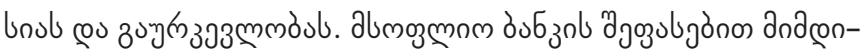

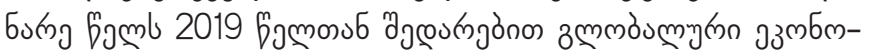

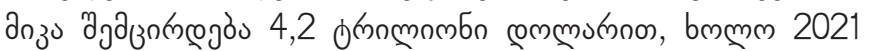

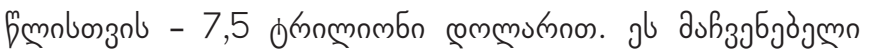

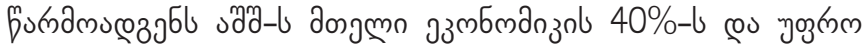

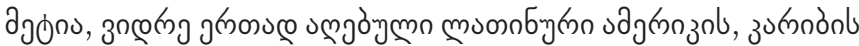

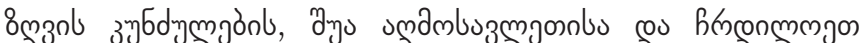

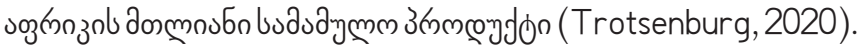

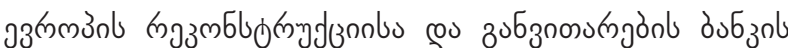

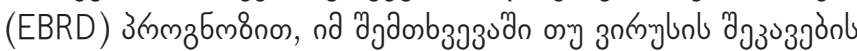

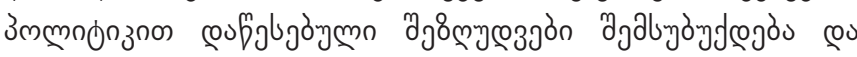

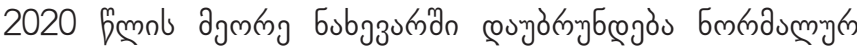

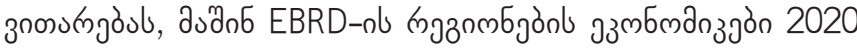

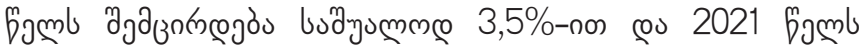

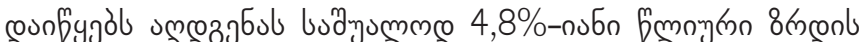

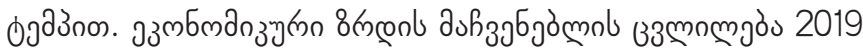

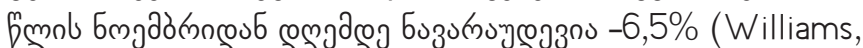

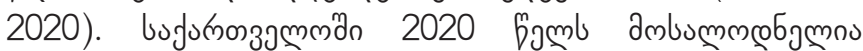

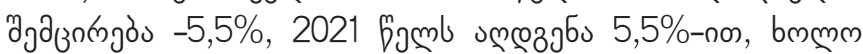

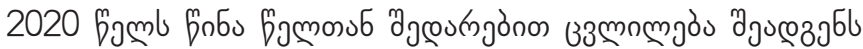
$-10 \%$ (EBRD, 2020).

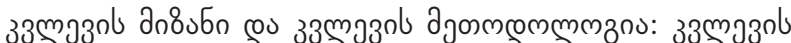

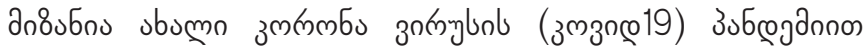
дез

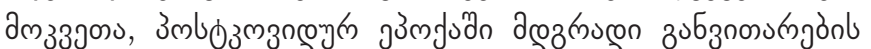

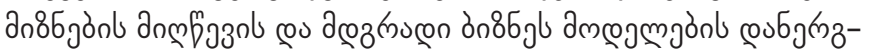

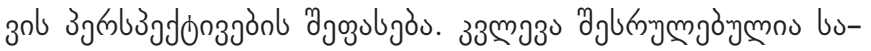

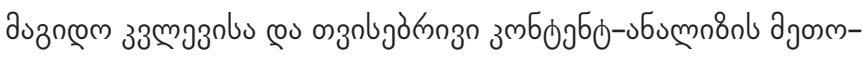
œgònl zuamyjбgònon.

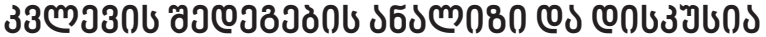

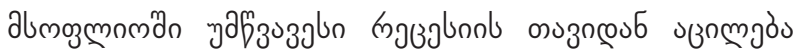

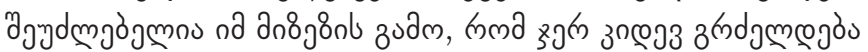

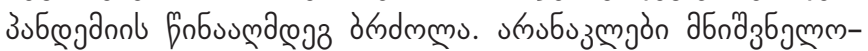
on samzubub formamuœ

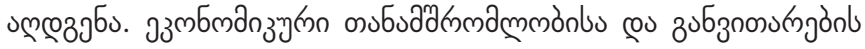

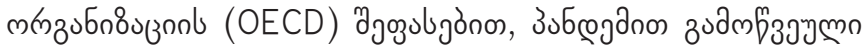

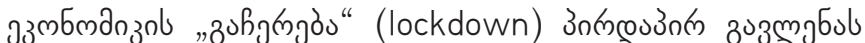

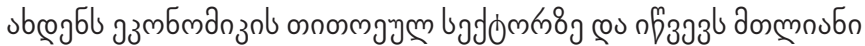

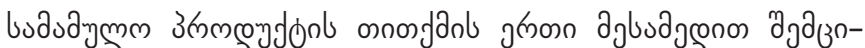

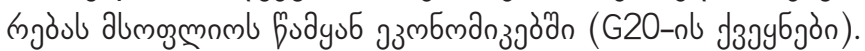

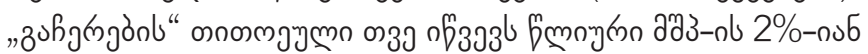

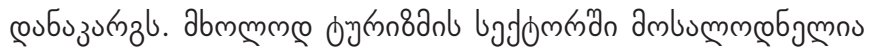

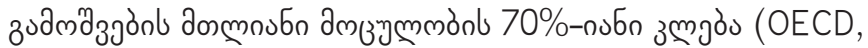

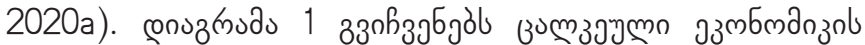

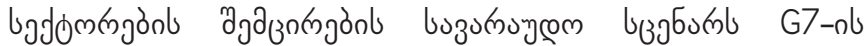

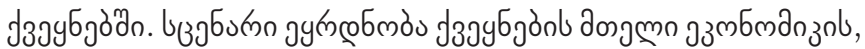

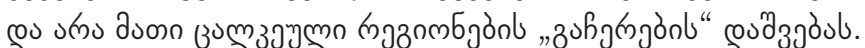

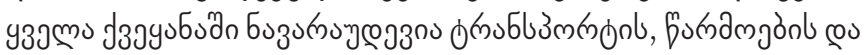

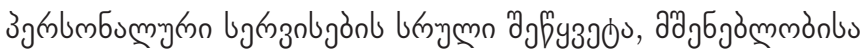

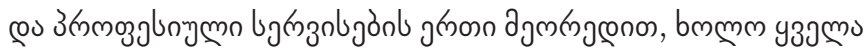

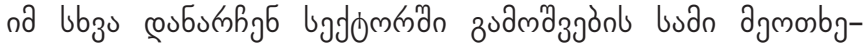

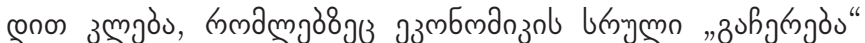

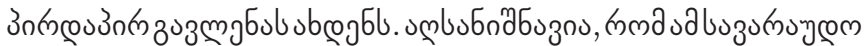

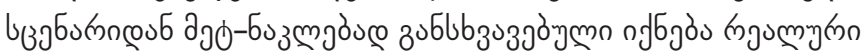

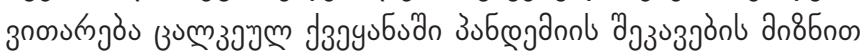

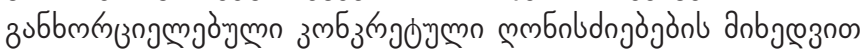
(OECD, 2020a). 


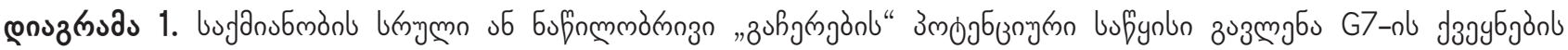
эзмбmanz 300

\section{аәз ауюодоз озьјвао ( \%)}

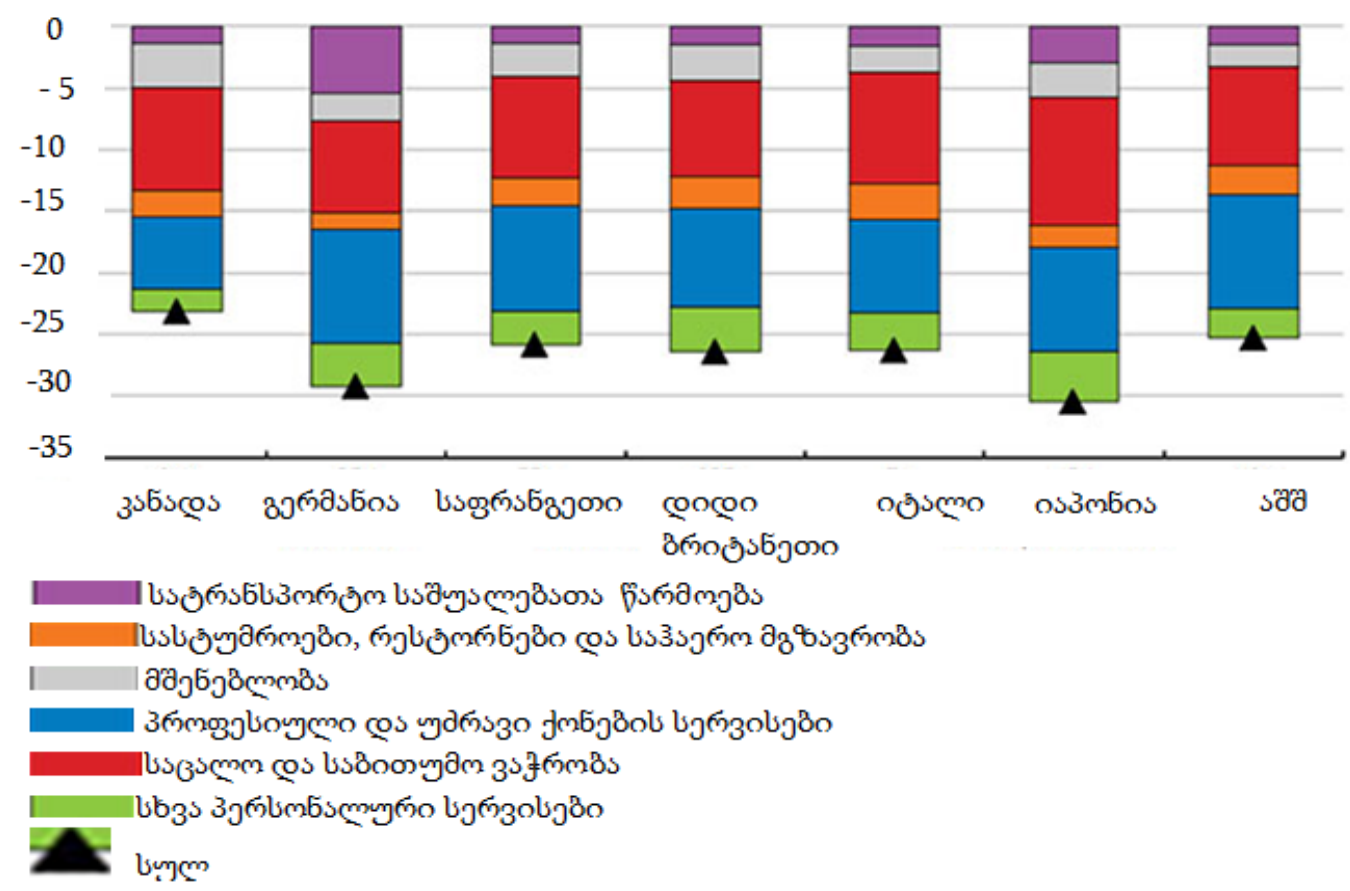

fyutrm: Organisation for Economic Co-operation and Development. http://www.oecd.org/hewsroom/oecd-updatesg20-summit-on-outlook-for-global-economy.htm

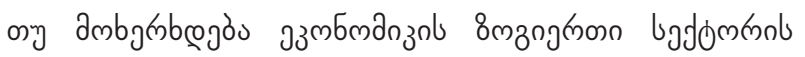

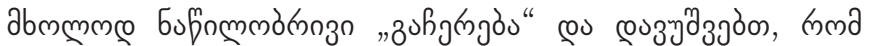

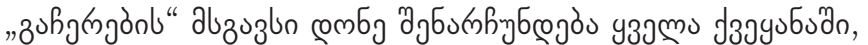

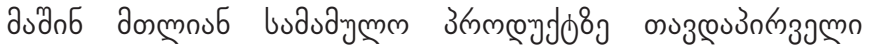

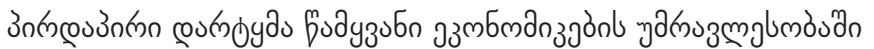

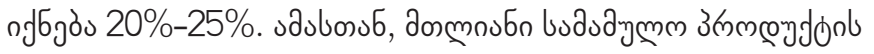

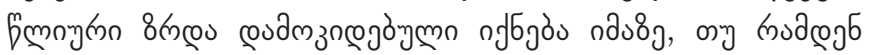

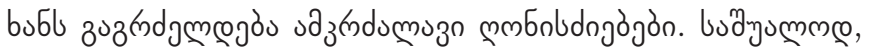

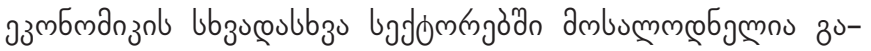

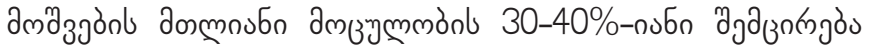

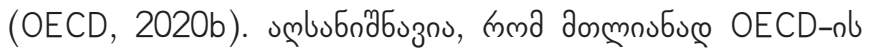

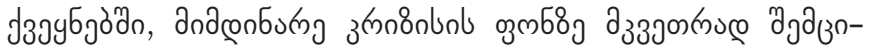

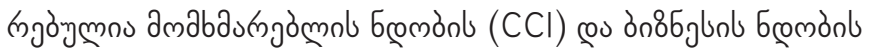

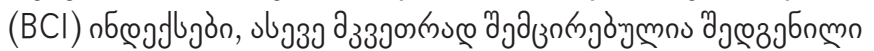

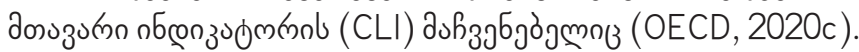

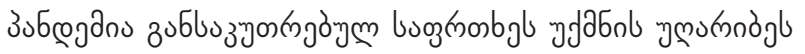

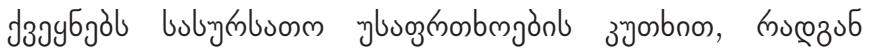

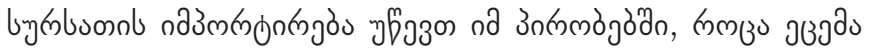

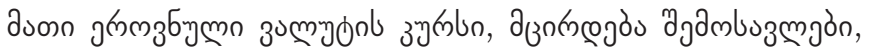

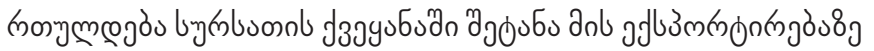

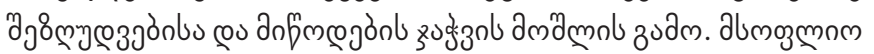

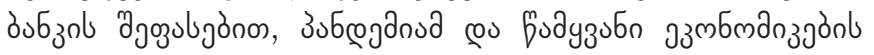

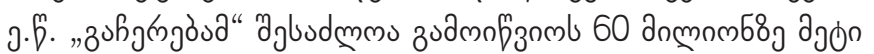

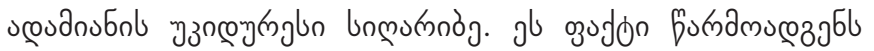

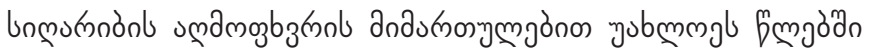

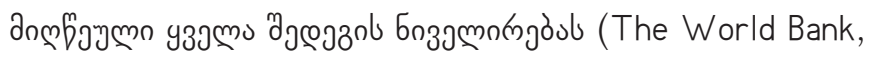
2020a).

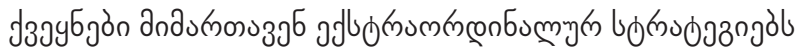

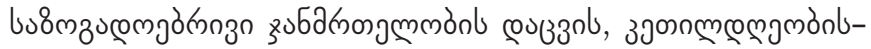

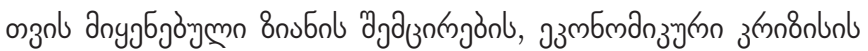

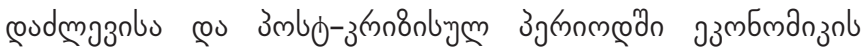

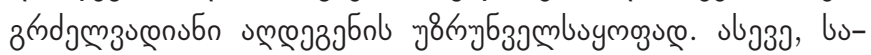

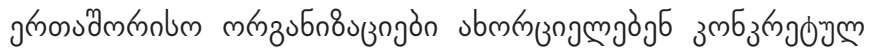

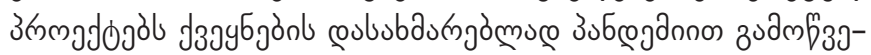

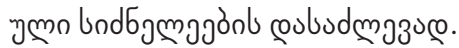

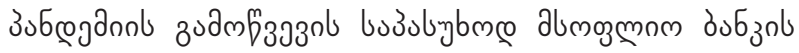

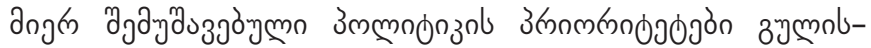

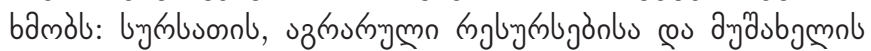

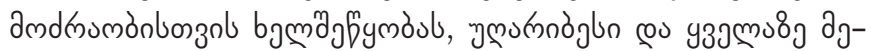

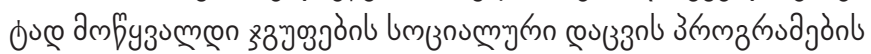

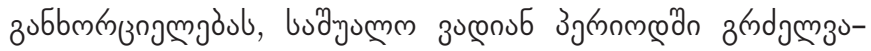

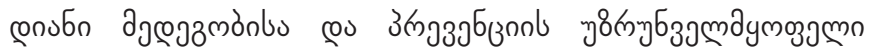

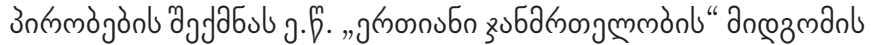

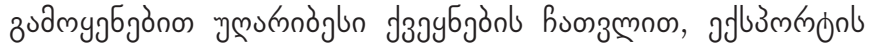

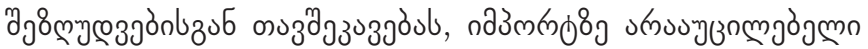

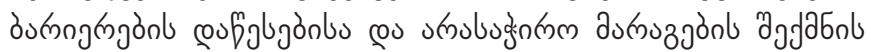

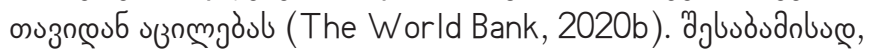

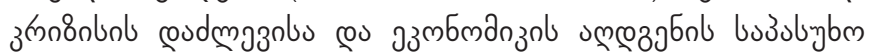

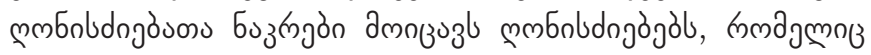

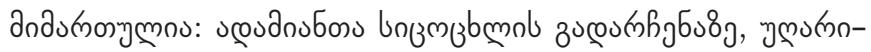




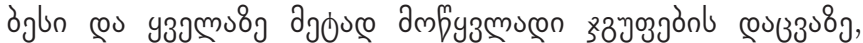

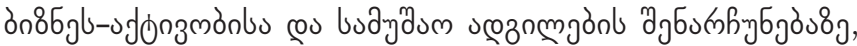

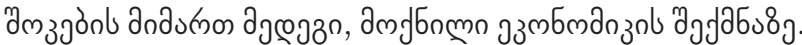

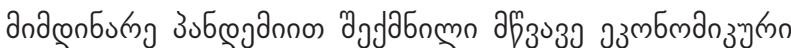

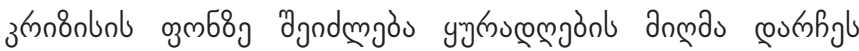

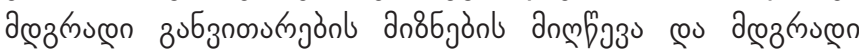

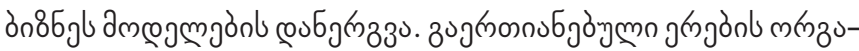

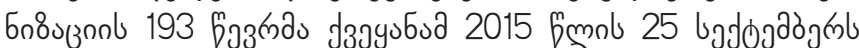

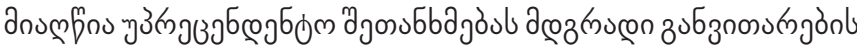

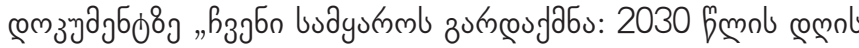

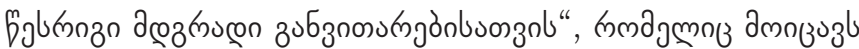
17 an8ubl cos 169 sam(zubul (United Nations, 2020).

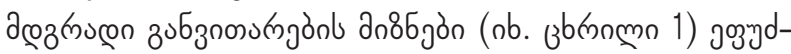

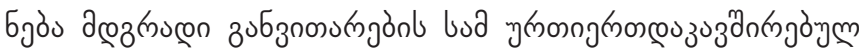

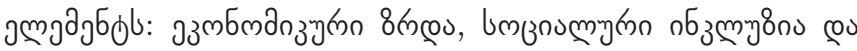

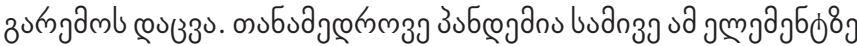

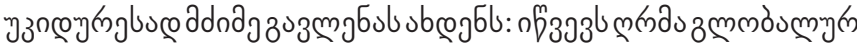

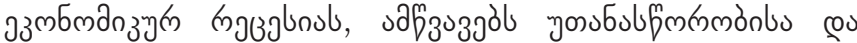

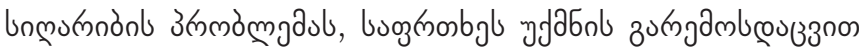

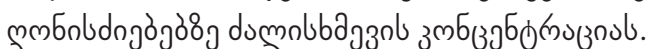

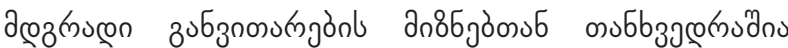

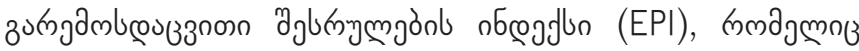

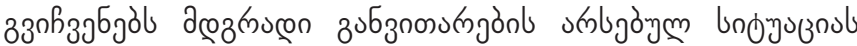

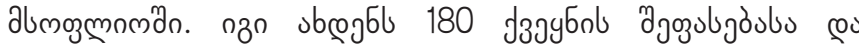

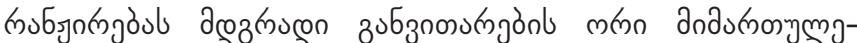

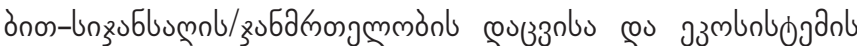

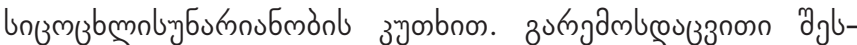

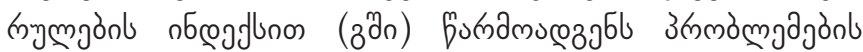
3ublu8ваз

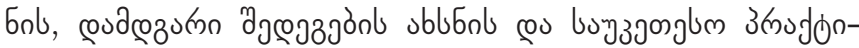

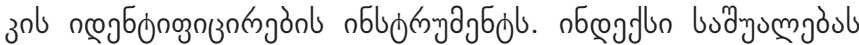

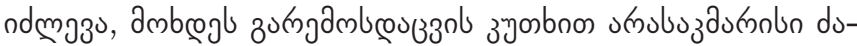

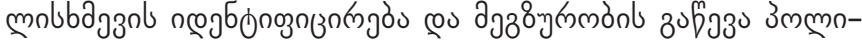

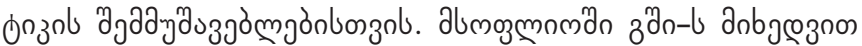

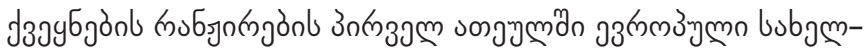

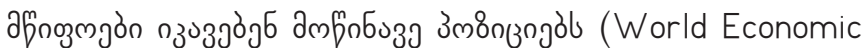
Forum, 2020a).

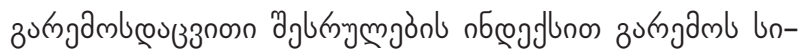

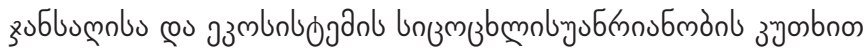

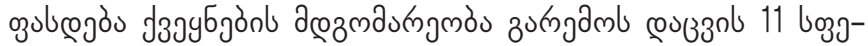

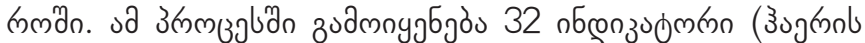

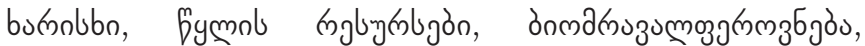

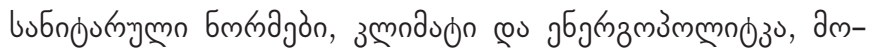

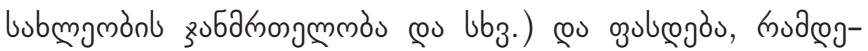

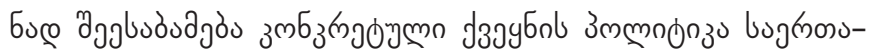

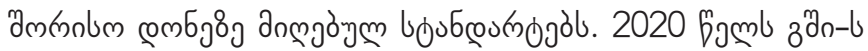

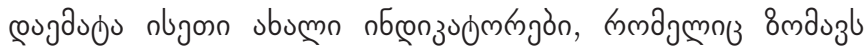

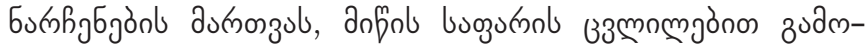

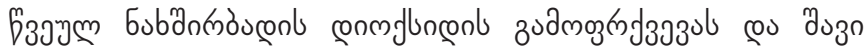

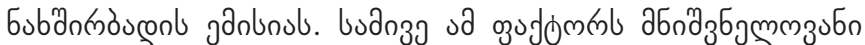

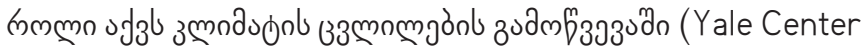
for Environmental Law and Policy, 2020). zumgambeouzznon

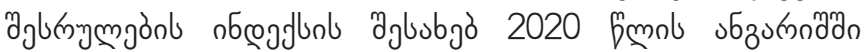

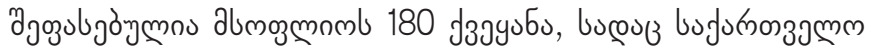

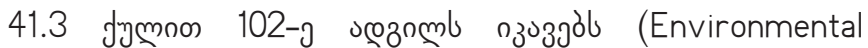
Performance Index, 2020a). zumgamb cou(3zol ümmnonzubons

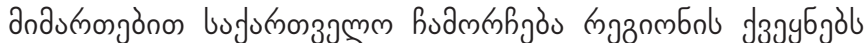

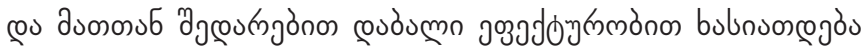
(Environmental Performance Index, 2020b). (nmzming

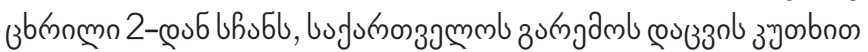

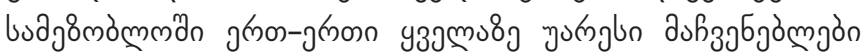

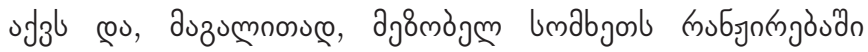

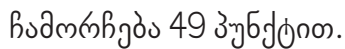

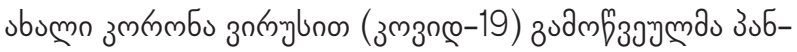

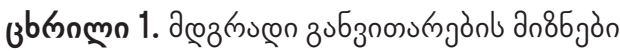

\begin{tabular}{|c|c|c|c|}
\hline \multicolumn{4}{|c|}{ 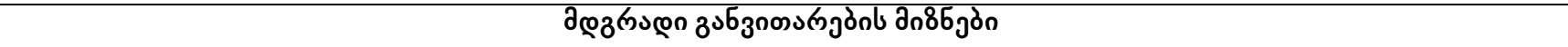 } \\
\hline 208งbn 1 & 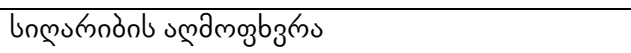 & 208ง6n 10 & 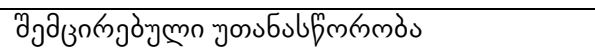 \\
\hline วо8งธก 2 & 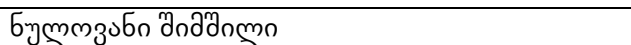 & วก8ง6n 11 & 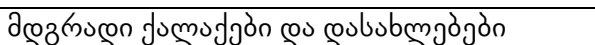 \\
\hline 208050 3 & 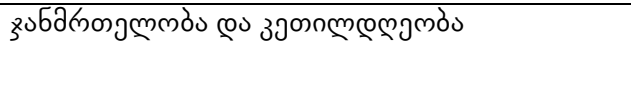 & 208ง6n 12 & 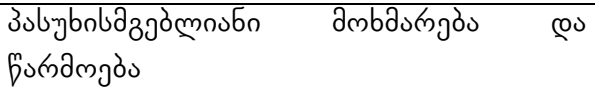 \\
\hline 2ถ8ง6n 4 & 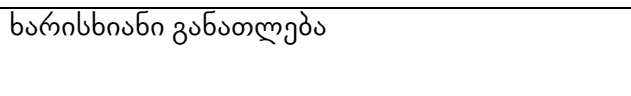 & 208ง6n 13 & 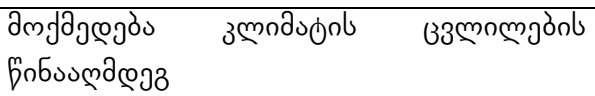 \\
\hline 2о8งธก 5 & 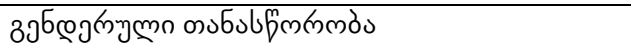 & 2о8ง6n 14 & 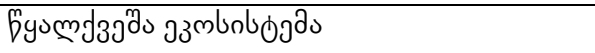 \\
\hline 2о8งbo 6 & byogos fyomo go bubndomos & 2о8ง6n 15 & anfob эзmbnbogas \\
\hline 2о8งธธก 7 & 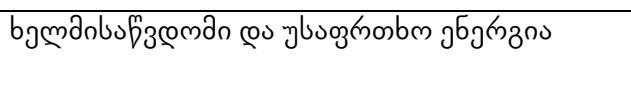 & วก8ง6n 16 & 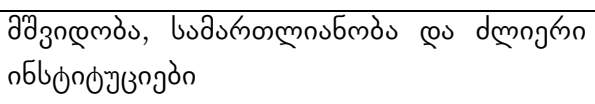 \\
\hline 2080508 & 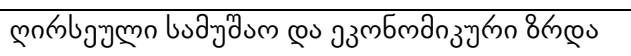 & 2о8งbn 17 & 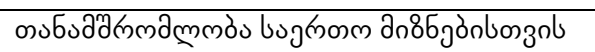 \\
\hline 2о8งธธก 9 & 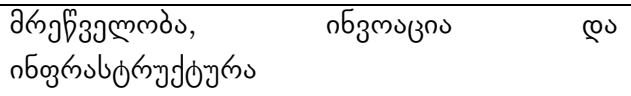 & & \\
\hline
\end{tabular}

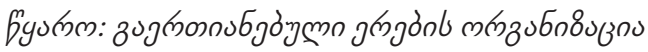




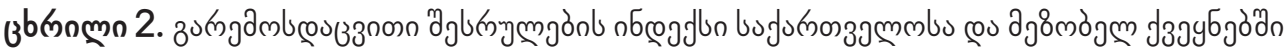

\begin{tabular}{|c|c|c|c|}
\hline d3agsobs & 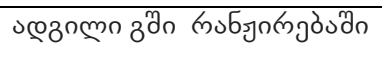 & $3^{2 n}$ لปmmo & 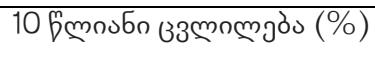 \\
\hline bufuknos 3amm & 102 & 41,3 & $-1,3$ \\
\hline कचलndgon & 99 & 42,6 & 2,1 \\
\hline 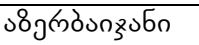 & 72 & 46 & 4 \\
\hline nybjom & 58 & 50,5 & 3,9 \\
\hline ঢmabjonn & 53 & 52,3 & 4,5 \\
\hline
\end{tabular}

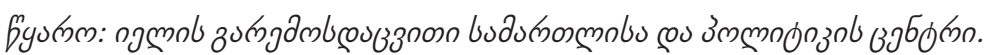

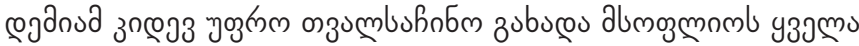

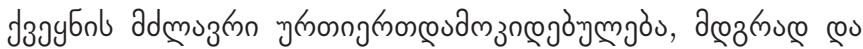

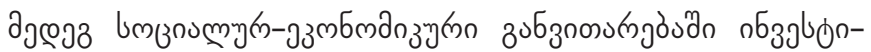

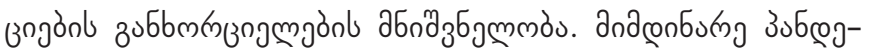

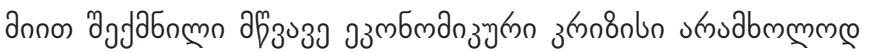

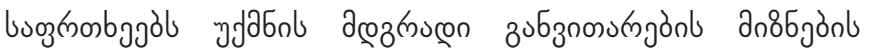

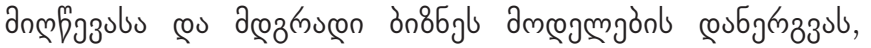

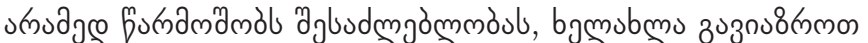

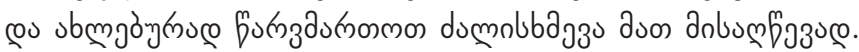

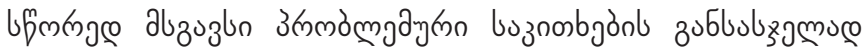

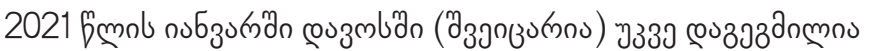

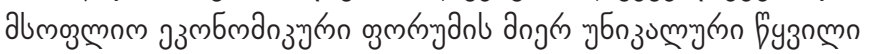

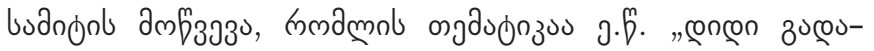

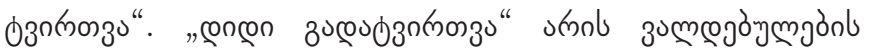

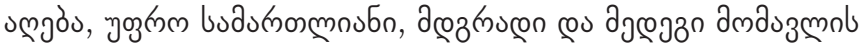

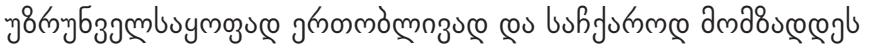

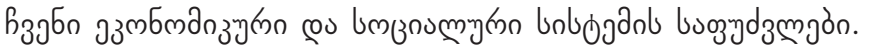

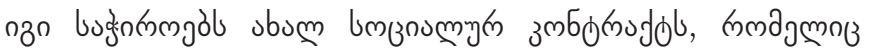

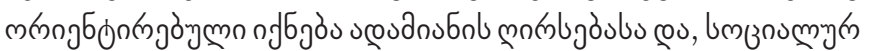
buauknom mn

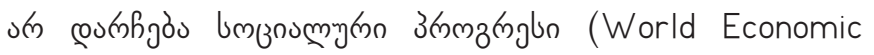
Forum, 2020b).

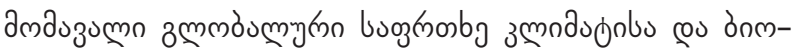
añzu mojg

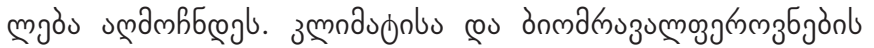

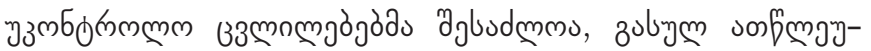

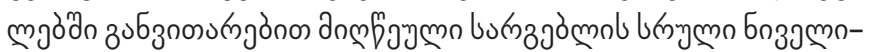

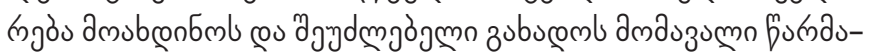

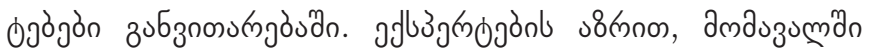

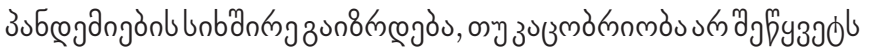

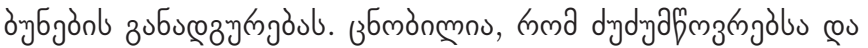

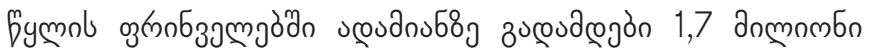

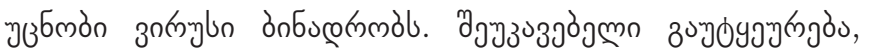

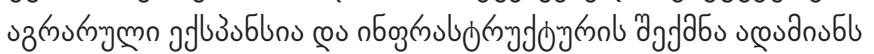

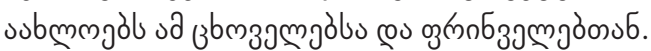

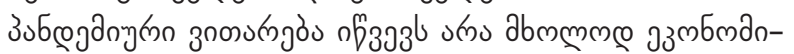

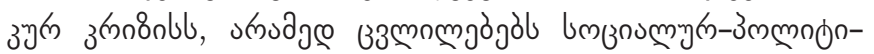

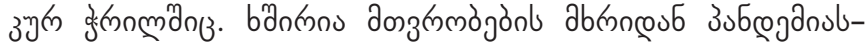

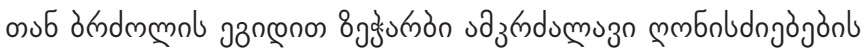

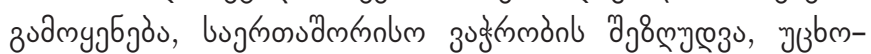

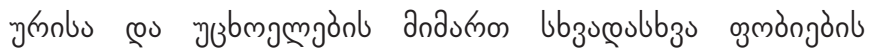

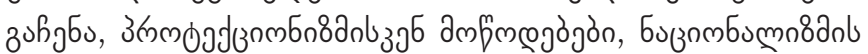

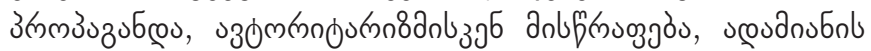

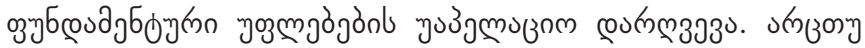

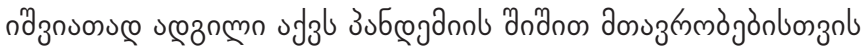

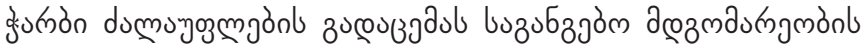

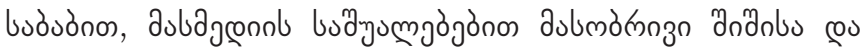

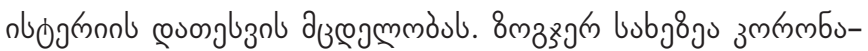

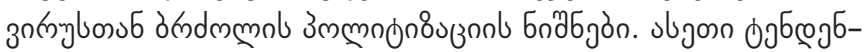
उ उ एо змзпюпвдn.

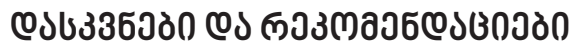

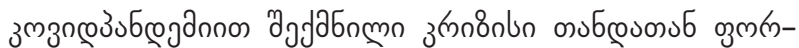

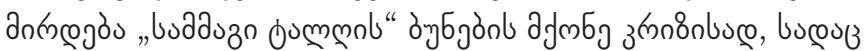

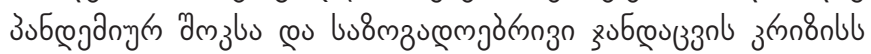

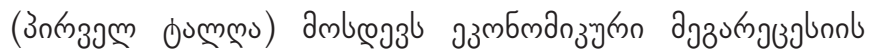

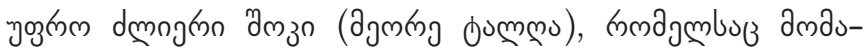

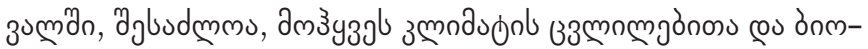

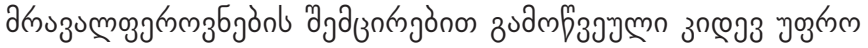

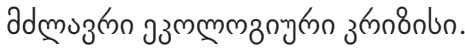

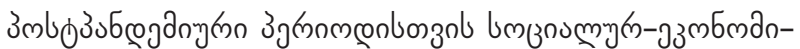

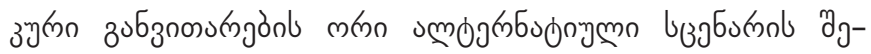

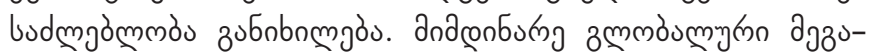

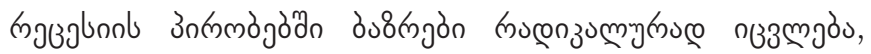

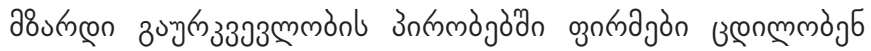

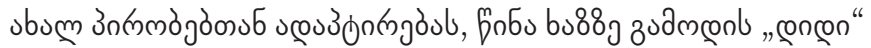

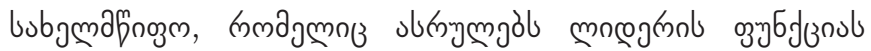

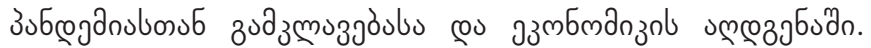

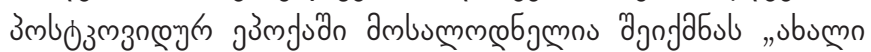

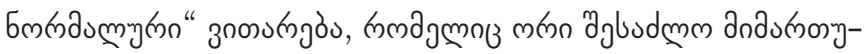

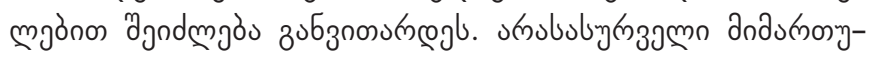

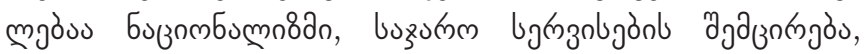




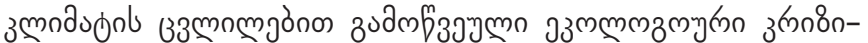

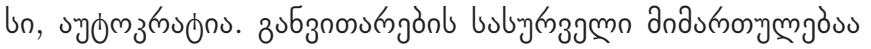
चलुभm

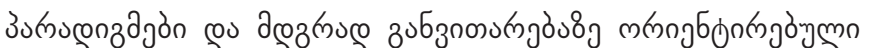

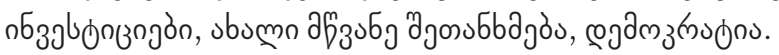

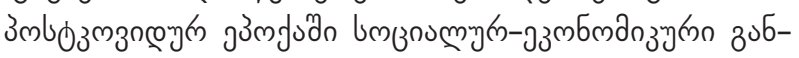

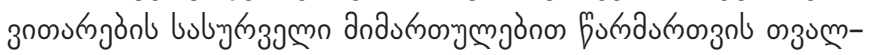

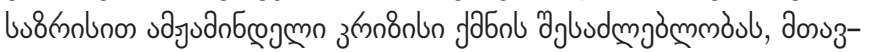

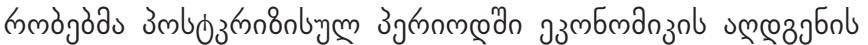

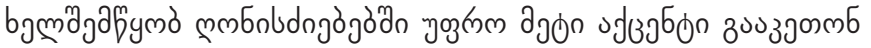

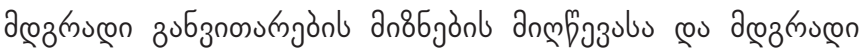

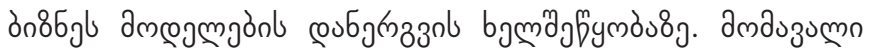

उub

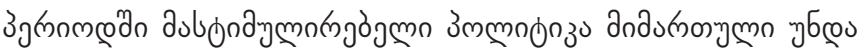

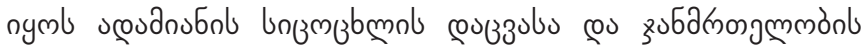

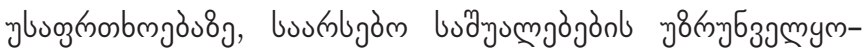

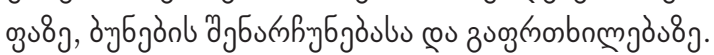

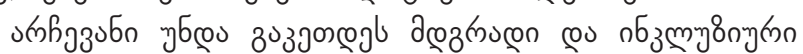

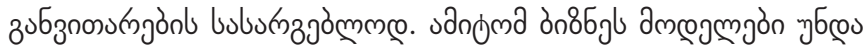

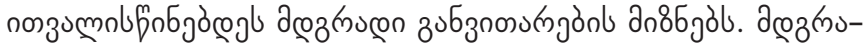

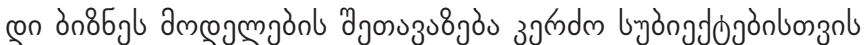

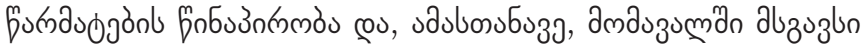
z

\section{REFERRENCES}

Environmental Performance Index 2020 (2020a). [online] https://epi.yale.edu/epi-results/2020/country/geo). Accessed on May 20, 2020.

Environmental Performance Index 2020 (2020b). [online] https://epi.yale.edu/epi-results/2020/component/epi). Accessed on May 20, 2020.

European Bank for Reconstruction and Development (2020). Regional Economic Prospects in the EBRD Regions. May 2020 update. Covid-19: From shock to recovery. [online] https://www.ebrd.com/what-we-do/economic-research-and-data/ rep.html Accessed on May 20, 2020.

Organisation for Economic Co-operation and Development (2020a). OECD updates G20 summit on outlook for global economy. 27/03/2020 - Updated 15 April 2020. [online] http://www.oecd.org/newsroom/oecd-updates-g20-summit-on-outlookfor-global-economy.htm) Accessed on May 20, 2020.

Organisation for Economic Co-operation and Development (2020b). Evaluating the initial impact of COVID-19 containment measures on economic activity. Updated 10 June 2020. [online] https://read.oecd-ilibrary.org/view/?ref=126_126496evgsi2gmqj\&title=Evaluating_the_initial_impact_of_COVID-19_containment_measures_on_economic_activity Accessed on June 20, 2020.

Organisation for Economic Co-operation and Development (2020c). Main Economic Indicators., Vol. 2020, Issue 6, June [online] https://read.oecd-ilibrary.org/economics/main-economic-indicators/volume-2020/issue-6_06aa372cen\#page5 Accessed on June 20, 2020.

The United Nations, Georgia (2020). Sustainable Development Goals. [online] https://georgia.un.org/sdgs\#.XsqYmkQzbIU Accessed on May 10, 2020.

The World Bank (2020a). World Bank Group: 100 Countries Get Support in Response to COVID-19 (Coronavirus)., 19 May, 2020. [online] https://www.worldbank.org/en/news/press-release/2020/05/19/world-bank-group-100-countriesget-support-in-response-to-covid-19-coronavirus?cid=ECR_E_NewsletterWeekly_EN_EXT\&deliveryName=DM65219 Accessed on May 20, 2020.

The World Bank (2020b). Statement by Mari Pangestu, Managing Director for Development Policy and Partnerships, The World Bank at the Virtual Meeting of G20 Agriculture Ministers. April 21, 2020. [online] https://www.worldbank.org/en/ news/speech/2020/04/21/statement-by-mari-pangestu-managing-director-for-development-policy-and-partnershipsthe-world-bank-at-the-virtual-meeting-of-g20-agriculture-ministers Accessed on May 20, 2020.

Trotsenburg, A. V., (2020) Broad, fast action to save lives and help countries rebuild. 06 May, 2020. [online] https://blogs. worldbank.org/voices/broad-fast-action-save-lives-and-help-countries rebuild?cid=ECR_E_NewsletterWeekly_EN_ EXT\&deliveryName=DM63224 Accessed on May 20, 2020.

Williams, A. (2020). EBRD economies seen contracting by 3.5 per cent in 2020, 4.8 per cent rebound in 2021. 13 May, 2020 [online] (https://www.ebrd.com/news/2020/ebrd-economies-seen-contracting-by-35-per-cent-in-2020-48-per-centrebound-in-2021.html Accessed on June 20, 2020.

World Economic Forum (2020a). This is the state of sustainability around the world. 12 June, 2020. [online] https://www. weforum.org/agenda/2020/06/chart-of-the-day-this-is-the-state-of-sustainability-around-the-world?fbclid=IwAROVNO ziYsATwDwSMhCU2IzXVzm8IFRy58tUpsRYWcnk-On6hlBCtZf_hbw Accessed on June 20, 2020.

World Economic Forum (2020b). The Great Reset: A Unique Twin Summit to Begin 2021. [online] https://www.weforum.org/ great-reset/about (Accessed on June 30, 2020).

Yale Center for Environmental Law and Policy (2020). Environmental Performance Index 2020. [online] https://envirocenter. yale.edu/2020-environmental-performance-index (Accessed on June 20, 2020). 